\title{
Periodicities in the Daily Proton Fluxes from 2011 to 2019 Measured by the Alpha Magnetic Spectrometer on the International Space Station from 1 to 100 GV
}

M. Aguilar, ${ }^{29}$ L. Ali Cavasonza, ${ }^{1}$ G. Ambrosi,${ }^{36}$ L. Arruda, ${ }^{27}$ N. Attig, ${ }^{24}$ F. Barao, ${ }^{27}$ L. Barrin, ${ }^{15}$ A. Bartoloni, ${ }^{42}$ S. Başeğmez-du Pree, ${ }^{18, *}$ R. Battiston, ${ }^{39,40}$ M. Behlmann, ${ }^{10}$ B. Beranek, ${ }^{1}$ J. Berdugo, ${ }^{29}$ B. Bertucci, ${ }^{36,37}$ V. Bindi, ${ }^{20}$ K. Bollweg, ${ }^{21}$ B. Borgia, ${ }^{42,43}$ M. J. Boschini, ${ }^{31}$ M. Bourquin, ${ }^{16}$ E. F. Bueno, ${ }^{18}$ J. Burger ${ }^{10}$ W. J. Burger ${ }^{39}$ S. Burmeister, ${ }^{25}$ X. D. Cai, ${ }^{10}$ M. Capell, ${ }^{10}$ J. Casaus, ${ }^{29}$ G. Castellini, ${ }^{14}$ F. Cervelli, ${ }^{38}$ Y. H. Chang, ${ }^{47}$ G. M. Chen, ${ }^{6,7}$ G. R. Chen, ${ }^{23}$ H. S. Chen, ${ }^{6,7}$ Y. Chen, ${ }^{16,23}$ L. Cheng, ${ }^{23}$ H. Y. Chou, ${ }^{47}$ S. Chouridou, ${ }^{1}$ V. Choutko, ${ }^{10}$ C. H. Chung, ${ }^{1}$ C. Clark, ${ }^{10,21}$ G. Coignet, ${ }^{3}$ C. Consolandi, ${ }^{20}$ A. Contin,${ }^{8,9}$ C. Corti, ${ }^{20}$ Z. Cui ${ }^{22,23}$ K. Dadzie, ${ }^{10}$ A. Dass,,${ }^{39,40}$ C. Delgado, ${ }^{29}$ S. Della Torre, ${ }^{31}$ M. B. Demirköz, ${ }^{2}$ L. Derome, ${ }^{17}$ S. Di Falco,${ }^{38}$ V. Di Felice,${ }^{44,}$ C. Díaz,${ }^{29}$ F. Dimiccoli, ${ }^{39}$ P. von Doetinchem, ${ }^{20}$ F. Dong,${ }^{34}$ F. Donnini, ${ }^{44, \dagger}$ M. Duranti, ${ }^{36}$ A. Egorov,${ }^{10}$ A. Eline,${ }^{10}$ J. Feng, ${ }^{10}$ E. Fiandrini,${ }^{36,37}$ P. Fisher, ${ }^{10}$ V. Formato, ${ }^{44,}$ C. Freeman,${ }^{20}$ C. Gámez ${ }^{29}$ R. J. García-López, ${ }^{26}$ C. Gargiulo, ${ }^{15}$ H. Gast,${ }^{1}$ M. Gervasi, ${ }^{31,32}$ F. Giovacchini, ${ }^{29}$ D. M. Gómez-Coral, ${ }^{20}$ J. Gong, ${ }^{34}$ C. Goy, ${ }^{3}$ V. Grabski, ${ }^{30}$ D. Grandi, ${ }^{31,32}$ M. Graziani, ${ }^{36,37}$ S. Haino, ${ }^{47}$ K. C. Han,${ }^{28}$ R. K. Hashmani, ${ }^{2}$ Z. H. He, ${ }^{19}$ B. Heber, ${ }^{25}$ T. H. Hsieh, ${ }^{10}$ J. Y. Hu, ${ }^{6,7}$ M. Incagli, ${ }^{38}$ W. Y. Jang, ${ }^{13}$ Yi Jia, ${ }^{10}$ H. Jinchi, ${ }^{28}$ G. Karagöz, ${ }^{2}$ B. Khiali, ${ }^{44, \dagger}$ G. N. Kim, ${ }^{13}$ Th. Kirn, ${ }^{1}$ M. Konyushikhin, ${ }^{10}$ O. Kounina, ${ }^{10}$ A. Kounine, ${ }^{10}$ V. Koutsenko, ${ }^{10}$ D. Krasnopevtsev, ${ }^{10}$ A. Kuhlman,${ }^{20}$ A. Kulemzin, ${ }^{10}$ G. La Vacca, ${ }^{31,32}$ E. Laudi, ${ }^{15}$ G. Laurenti, ${ }^{8}$ I. Lazzizzera, ${ }^{39,40}$ A. Lebedev, ${ }^{10}$ H. T. Lee, ${ }^{46}$ S. C. Lee, ${ }^{47}$ J. Q. Li, ${ }^{34}$ M. Li, ${ }^{1}$ Q. Li, ${ }^{34}$ S. Li, ${ }^{1}$ J. H. Li ${ }^{22}$ Z. H. Li,${ }^{67}$ J. Liang, ${ }^{22}$ C. Light,${ }^{20}$ C. H. Lin, ${ }^{47}$ T. Lippert, ${ }^{24}$ J. H. Liu, ${ }^{5}$ Z. Liu, ${ }^{16}$ S. Q. Lu, ${ }^{47}$ Y. S. Lu, ${ }^{6}$ K. Luebelsmeyer, ${ }^{1}$ J. Z. Luo, ${ }^{34}$ Xi Luo, ${ }^{23}$ F. Machate, ${ }^{1}$ C. Mañá, ${ }^{29}$ J. Marín, ${ }^{29}$ J. Marquardt, ${ }^{25}$ T. Martin, ${ }^{10,21}$ G. Martínez, ${ }^{29}$ N. Masi,${ }^{8,9}$ D. Maurin, ${ }^{17}$ T. Medvedeva, ${ }^{10}$ A. Menchaca-Rocha, ${ }^{30}$ Q. Meng, ${ }^{34}$

V. V. Mikhailov, ${ }^{33}$ M. Molero, ${ }^{29}$ P. Mott, ${ }^{10,21}$ L. Mussolin, ${ }^{36,37}$ J. Negrete, ${ }^{20}$ N. Nikonov, ${ }^{1}$ F. Nozzoli, ${ }^{39}$ A. Oliva, ${ }^{8}$ M. Orcinha, ${ }^{27}$ M. Palermo, ${ }^{20}$ F. Palmonari, ${ }^{8,9}$ M. Paniccia, ${ }^{16}$ A. Pashnin, ${ }^{10}$ M. Pauluzzi, ${ }^{36,37}$ S. Pensotti, ${ }^{31,32}$ H. D. Phan, ${ }^{10}$ V. Plyaskin, ${ }^{10}$ M. Pohl,${ }^{16}$ S. Poluianov, ${ }^{35}$ X. Qin, ${ }^{10}$ Z. Y. Qu, ${ }^{47}$ L. Quadrani, ${ }^{8,9}$ P. G. Rancoita, ${ }^{31}$ D. Rapin, ${ }^{16}$

A. Reina Conde, ${ }^{26}$ E. Robyn, ${ }^{16}$ S. Rosier-Lees, ${ }^{3}$ A. Rozhkov, ${ }^{10}$ D. Rozza, ${ }^{31,32}$ R. Sagdeev, ${ }^{11}$ S. Schael,,${ }^{1}$ A. Schultz von Dratzig, ${ }^{1}$ G. Schwering, ${ }^{1}$ E. S. Seo, ${ }^{12}$ Z. Shakfa, ${ }^{2}$ B. S. Shan, ${ }^{4}$ T. Siedenburg, ${ }^{1}$ C. Solano, ${ }^{10}$ J. W. Song, ${ }^{22}$ X. J. Song, ${ }^{23}$ R. Sonnabend, ${ }^{1}$ L. Strigari, ${ }^{42,}$ T. Su, ${ }^{23}$ Q. Sun, ${ }^{22}$ Z. T. Sun, ${ }^{6,7}$ M. Tacconi,${ }^{31,32}$ X. W. Tang, ${ }^{6}$ Z. C. Tang, ${ }^{6}$ J. Tian, ${ }^{36,37}$ Samuel C. C. Ting $\odot,{ }^{10,15}$ S. M. Ting, ${ }^{10}$ N. Tomassetti, ${ }^{36,37}$ J. Torsti, ${ }^{49}$ T. Urban,,${ }^{10,21}$ I. Usoskin, ${ }^{35}$ V. Vagelli, ${ }^{41,36}$ R. Vainio, ${ }^{49}$ M. Valencia-Otero, ${ }^{48}$ E. Valente ${ }^{42,43}$ E. Valtonen, ${ }^{49}$ M. Vázquez Acosta, ${ }^{26}$ M. Vecchi, ${ }^{18}$ M. Velasco, ${ }^{29}$ J. P. Vialle, ${ }^{3}$ C. X. Wang, ${ }^{22}$ L. Wang, ${ }^{5}$ L. Q. Wang, ${ }^{22}$ N. H. Wang, ${ }^{22}$ Q. L. Wang, ${ }^{5}$ S. Wang,${ }^{20}$ X. Wang, ${ }^{10}$ Yu Wang, ${ }^{22}$ Z. M. Wang, ${ }^{23}$ J. Wei, ${ }^{16}$ Z. L. Weng, ${ }^{10}$ H. Wu, ${ }^{34}$ R. Q. Xiong, ${ }^{34}$ W. Xu, ${ }^{22,23}$ Q. Yan, ${ }^{10}$ Y. Yang, ${ }^{45}$ I. I. Yashin, ${ }^{33}$ H. Yi,${ }^{34}$ Y. M. Yu, ${ }^{10}$ Z. Q. Yu, ${ }^{6}$ M. Zannoni, ${ }^{31,32}$ C. Zhang, ${ }^{6}$ F. Zhang, ${ }^{6}$ F. Z. Zhang, ${ }^{6,7}$ J. H. Zhang, ${ }^{34}$ Z. Zhang, ${ }^{10}$ F. Zhao, ${ }^{6,7}$ C. Zheng, ${ }^{23}$ Z. M. Zheng, ${ }^{4}$ H. L. Zhuang, ${ }^{6}$ V. Zhukov, ${ }^{1}$ A. Zichichi, ${ }^{8,9}$ and P. Zuccon ${ }^{39,40}$

(AMS Collaboration)

\footnotetext{
${ }^{1}$ I. Physics Institute and JARA-FAME, RWTH Aachen University, 52056 Aachen, Germany

${ }^{2}$ Department of Physics, Middle East Technical University (METU), 06800 Ankara, Turkey

${ }^{3}$ Université Grenoble Alpes, Université Savoie Mont Blanc, CNRS, LAPP-IN2P3, 74000 Annecy, France

${ }^{4}$ Beihang University (BUAA), Beijing, 100191, China

${ }^{5}$ Institute of Electrical Engineering (IEE), Chinese Academy of Sciences, Beijing, 100190, China

${ }^{6}$ Institute of High Energy Physics (IHEP), Chinese Academy of Sciences, Beijing, 100049, China

${ }^{7}$ University of Chinese Academy of Sciences (UCAS), Beijing, 100049, China

${ }^{8}$ INFN Sezione di Bologna, 40126 Bologna, Italy

${ }^{9}$ Università di Bologna, 40126 Bologna, Italy

${ }^{10}$ Massachusetts Institute of Technology (MIT), Cambridge, Massachusetts 02139, USA

${ }^{11}$ East-West Center for Space Science, University of Maryland, College Park, Maryland 20742, USA

${ }^{12}$ IPST, University of Maryland, College Park, Maryland 20742, USA

${ }^{13}$ CHEP, Kyungpook National University, 41566 Daegu, Korea

${ }^{14}$ CNR-IROE, 50125 Firenze, Italy

${ }^{15}$ European Organization for Nuclear Research (CERN), 1211 Geneva 23, Switzerland

${ }^{16}$ DPNC, Université de Genève, 1211 Genève 4, Switzerland

${ }^{17}$ Université Grenoble Alpes, CNRS, Grenoble INP, LPSC-IN2P3, 38000 Grenoble, France

${ }^{18}$ Kapteyn Astronomical Institute, University of Groningen, P.O. Box 800, 9700 AV Groningen, Netherlands
} 


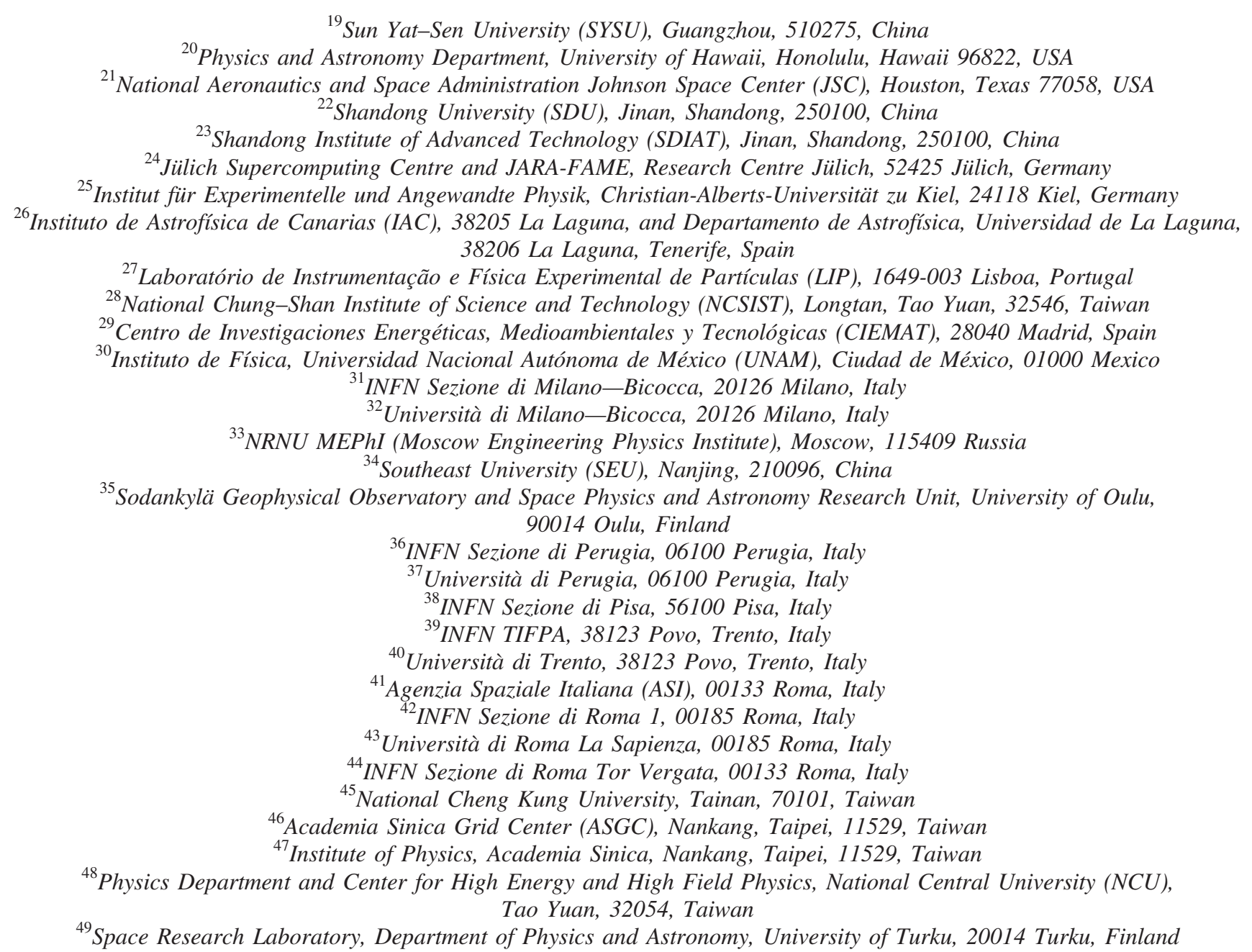

(Received 19 June 2021; revised 24 September 2021; accepted 8 November 2021; published 27 December 2021)

We present the precision measurement of the daily proton fluxes in cosmic rays from May 20, 2011 to October 29, 2019 (a total of 2824 days or 114 Bartels rotations) in the rigidity interval from 1 to $100 \mathrm{GV}$ based on $5.5 \times 10^{9}$ protons collected with the Alpha Magnetic Spectrometer aboard the International Space Station. The proton fluxes exhibit variations on multiple timescales. From 2014 to 2018, we observed recurrent flux variations with a period of 27 days. Shorter periods of 9 days and 13.5 days are observed in 2016 . The strength of all three periodicities changes with time and rigidity. The rigidity dependence of the 27-day periodicity is different from the rigidity dependences of 9-day and 13.5-day periods. Unexpectedly, the strength of 9-day and 13.5-day periodicities increases with increasing rigidities up to $\sim 10 \mathrm{GV}$ and $\sim 20 \mathrm{GV}$, respectively. Then the strength of the periodicities decreases with increasing rigidity up to $100 \mathrm{GV}$.

DOI: 10.1103/PhysRevLett.127.271102

The temporal evolution of the interplanetary space environment causes cosmic-ray intensity variations. This is particularly visible at energies below $100 \mathrm{GeV}$. These

Published by the American Physical Society under the terms of the Creative Commons Attribution 4.0 International license. Further distribution of this work must maintain attribution to the author(s) and the published article's title, journal citation, and DOI. variations correlate with solar activity at different timescales $[1,2]$. The most significant long-term scale variation is the 11-year solar cycle during which the number of sunspots changes from minimum to maximum and then back to a minimum [3,4]. Shorter scale variations can be either nonrecurrent or recurrent. The nonrecurrent variations are mainly due to the interactions of cosmic rays with strong transient disturbances in the interplanetary magnetic field, such as shock waves generated by interplanetary 
coronal mass ejections, especially during solar maxima, that can last from days to weeks [5]. Recurrent variations with a period of 27 days, corresponding to the synodic solar rotation, and at multiples of that frequency (e.g., periods of 13.5 and 9 days) are related to the passage of corotating interaction regions originating from one or more coronal holes of the Sun [6-14], as first observed in 1938 [15]. Previous studies on the estimated rigidity dependence in periodicities, for example in Ref. [11], generally concluded that the power of the periodicity decreases with increasing rigidity. This formed the paradigm over the AMS rigidity range (1 to $100 \mathrm{GV}$ ) that the strength of the 27-day (and 13.5-day, 9-day) periodicities steadily decreases with increasing rigidity of cosmic rays, differently in solar maximum and minimum [16].

Previously, the Alpha Magnetic Spectrometer (AMS) has reported the time dependence of proton and helium fluxes on the timescale of Bartels rotations (BR: 27 days) [17].

To date, there are no direct continuous daily measurements of the rigidity dependence of 9-day, 13.5-day, and 27-day periodicities over an extended period of time and a broad range of rigidities.

In this Letter, we present the daily time evolution of the proton flux from 1.00 to $100 \mathrm{GV}$. The measurement is based on $5.5 \times 10^{9}$ protons collected by AMS during the first 8.5 years (May 20, 2011 to October 29, 2019, a total of 2824 days or 114 BRs) of operation aboard the International Space Station (ISS).

Detector.-The layout and description of the AMS detector are presented in Refs. [18,19] and shown in Fig. S1 of the Supplemental Material (SM) [20]. The key elements used in this measurement are the permanent magnet [21], the silicon tracker [22-24], and the four planes of time of flight (TOF) scintillation counters [25]. Further information on the AMS layout, performance, trigger, and the Monte Carlo (MC) simulations [26,27] is detailed in the SM [20].

Event selection.-AMS has collected $1.5 \times 10^{11}$ cosmic ray events from May 20, 2011 to October 29, 2019. Proton events are required to be downward going and to have a reconstructed track in the inner tracker. See Fig. S2 of the SM [20] for a reconstructed proton event. Details of the event selection and backgrounds are contained in Refs. [17,28-31] and in the SM [20]. After selection, the event sample contains $5.5 \times 10^{9} Z=1$ particles. The sample includes a few percent of deuterons [32].

Data analysis.-The daily isotropic flux $\Phi_{i}^{j}$ in the $i$ th rigidity bin $\left(R_{i}, R_{i}+\Delta R_{i}\right)$ and $j$ th day is given by

$$
\Phi_{i}^{j}=\frac{N_{i}^{j}}{A_{i}^{j} \epsilon_{i}^{j} T_{i}^{j} \Delta R_{i}},
$$

where $N_{i}^{j}$ is the number of events corrected for bin-to-bin migration, $A_{i}^{j}$ is the effective acceptance, $\epsilon_{i}^{j}$ is the trigger efficiency, and $T_{i}^{j}$ is the daily collection time. In this Letter, the proton flux was measured in 30 bins from 1.00 to $100 \mathrm{GV}$. Bin-to-bin migration of events was corrected using the unfolding procedures described in Ref. [28] independently for each day.

Extensive studies were made of the systematic errors [28]. These errors include the uncertainties in the background evaluation, the trigger efficiency, the geomagnetic cutoff, the acceptance calculation, the rigidity resolution function, and the absolute rigidity scale.

The systematic error on the proton fluxes associated with the trigger efficiency measurement is $<1 \%$ over the entire rigidity range and for all days.

The calculated geomagnetic cutoff as described in the SM [20] was increased by $10 \%$ and the resulting systematic error in the fluxes is $<2 \%$ at $1 \mathrm{GV}$ and negligible $(<0.4 \%)$ above $2 \mathrm{GV}$.

The effective acceptances $A_{i}^{j}$ were calculated using MC simulation and corrected for small differences between the data and simulated events related to (a) event reconstruction and selection, namely, in the efficiencies of velocity vector determination, track finding, charge determination, and tracker quality cuts and (b) the details of inelastic interactions of protons in the AMS materials. The systematic error on the fluxes associated with the reconstruction and selection is $<1 \%$ over the entire rigidity range for all days. The time-independent systematic error on the proton fluxes due to uncertainties in the evaluation of the inelastic interactions is $<1 \%$ over the entire rigidity range [28].

The time-independent rigidity resolution function for protons has a pronounced Gaussian core and non-Gaussian tails. The systematic error on the fluxes due to the rigidity resolution function was obtained by repeating the unfolding procedure while independently varying the width of the Gaussian core by $5 \%$ and the amplitude of the nonGaussian tails by $20 \%$ [28]. The resulting systematic error on the fluxes is $1.5 \%$ at $1 \mathrm{GV}$ and $<1 \%$ above $2 \mathrm{GV}$.

There are two contributions to the systematic uncertainty on the rigidity scale [28]. The first is due to residual tracker misalignment. This error was estimated by comparing the $E / p$ ratio for electrons and positrons, where $E$ is the energy measured with the electromagnetic calorimeter and $p$ is the momentum measured with the tracker. It was found to be $1 / 30 \mathrm{TV}^{-1}$ [33]. The error is negligible $(<0.3 \%)$ below $100 \mathrm{GV}$. The second systematic error on the rigidity scale arises from the magnetic field map measurement and its temperature corrections. The total time-independent error on the fluxes due to uncertainty on the rigidity scale has been calculated to be $<0.6 \%$ over the rigidity range below $100 \mathrm{GV}$.

The contributions to the systematic error from the trigger and reconstruction efficiencies are evaluated independently each day and are added in quadrature to derive a timedependent systematic error, which is $<2 \%$ at $1 \mathrm{GV}$ and $<1 \%$ above $2 \mathrm{GV}$ for all days. The daily total systematic 
error is obtained by adding in quadrature the individual contributions of the time-independent systematic errors discussed above and the time-dependent systematic errors. At $1 \mathrm{GV}$ it is $<3.4 \%$ and above $2 \mathrm{GV}$ it is $<1.4 \%$ for all days.

Most importantly, several independent analyses were performed on the same data sample by different study groups. The results of those analyses are consistent with this Letter.

Results.-The measured daily proton fluxes including statistical errors, time-dependent systematic errors, and total systematic errors are tabulated in Tables S1-S2824 of SM [20] as functions of the rigidity at the top of the AMS detector. For the days when AMS detected solar energetic particles (SEPs), the fluxes below $3 \mathrm{GV}$ will be included in a future publication [34] and not here.

Figure 1 shows the daily proton fluxes for six rigidity bins from 1.00 to $10.10 \mathrm{GV}$. In this and subsequent figures, the error bars on the fluxes are the sum in quadrature of the statistical and time-dependent systematic errors. As seen, the proton fluxes exhibit variations on different timescales, from days to years. The relative magnitude of these

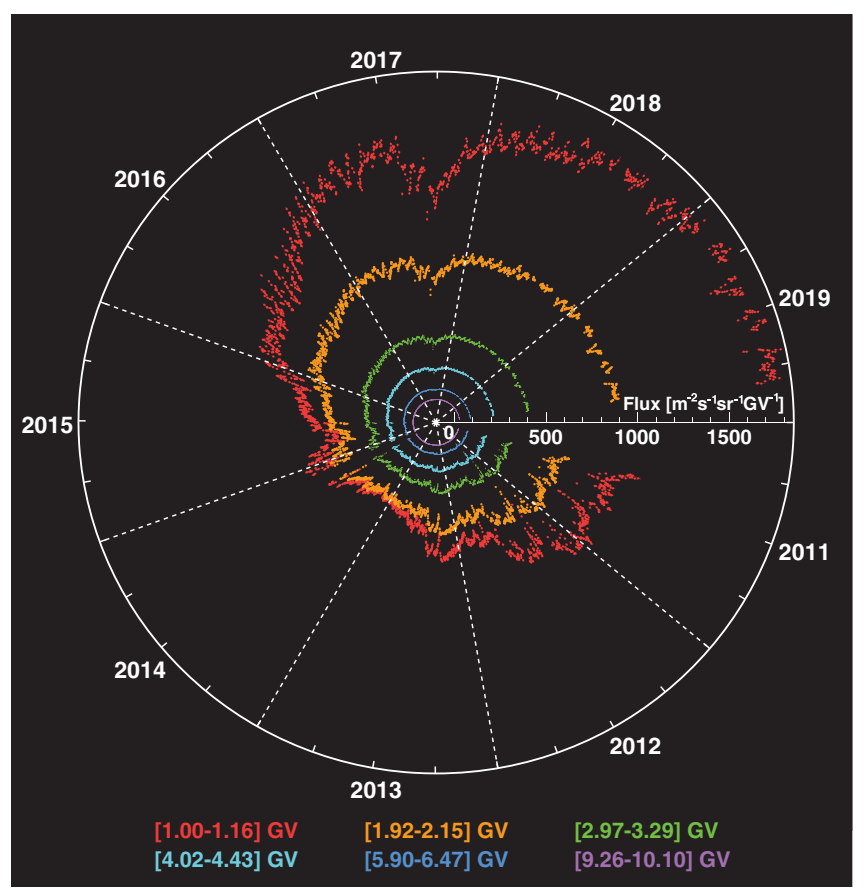

FIG. 1. The daily AMS proton fluxes for six typical rigidity bins from 1.00 to $10.10 \mathrm{GV}$ measured from May 20, 2011 to October 29, 2019 which includes a major portion of solar cycle 24 (from December 2008 to December 2019). The AMS data cover the ascending phase, the maximum, and descending phase to the minimum of solar cycle 24. Days with SEPs are removed for the two lowest rigidity bins. The gaps in the fluxes are due to detector studies and upgrades. The error bars are invisible. As seen, the proton fluxes exhibit large variations with time, and the relative magnitude of these variations decreases with increasing rigidity. variations decreases with increasing rigidity. At low rigidities, recurrent flux variations are clearly visible. An explanation of the dip in 2017 is presented in the SM [20]. Figures S3 and S4 of the SM [20] show the comparison of AMS daily proton fluxes with results from PAMELA [35].

Figure 2 shows the daily AMS proton fluxes measured in 2016 for three rigidity bins [1.00-1.16] GV, [5.906.47] GV, and [16.60-22.80] GV. As seen, double-peak and triple-peak structures are visible in different Bartels rotations.

To study the recurrent time variations in the daily proton fluxes, a wavelet time-frequency technique $[36,37]$ was used to locate the time intervals where the periodic structures emerge. The details on the wavelet analysis are described in the SM [20]. All the power spectra in the subsequent figures of the text and of the SM [20] are drawn with normalized power defined in the SM [20] to show the strength of the periodicities. The daily proton fluxes for the same three rigidity bins in each of the nine years (2011-2019), together with their time-averaged power spectra and 95\% confidence levels, are shown in Figs. S5-S13 of the SM [20]. We observed recurrent flux variations with a period of 27 days with significance above the $95 \%$ confidence level from 2014 to 2018. Shorter periods of $\sim 13.5$ days and $\sim 9$ days are significant only in 2016.

To study the details of periodicity in 2016, Fig. 3 shows the wavelet time-frequency power spectra of daily proton fluxes for the same three rigidity bins. As seen, periods of 9 , 13.5, and 27 days are observed at different time intervals. The strength of all three periodicities changes with time and rigidity. In particular, shorter periods of 9 and 13.5 days, when present, are more visible at $6 \mathrm{GV}$ and $20 \mathrm{GV}$ compared to $1 \mathrm{GV}$. We define two time intervals of interest marked on the top of Fig. 3: the first time interval (BRs 2489-2495) is when the 9-day period is visible; the second time interval (BRs 2496-2502) is when the 9-day period is not visible.

Figure 4 shows the normalized power as a function of rigidity and period for the two time intervals. As seen, the strength of all three periodicities is rigidity dependent. In particular, the strength of 9-day and 13.5-day periodicities increases with increasing rigidity up to $\sim 10 \mathrm{GV}$ and $\sim 20 \mathrm{GV}$, respectively, and then decreases with increasing rigidity up to $100 \mathrm{GV}$. Thus, the AMS results do not support the general conclusion that the strength of the periodicities steadily decreases with increasing rigidity. The projections of the normalized power on the period and on the rigidity together with the $95 \%$ confidence levels are included in Fig. S14 and Fig. S15 of the SM [20], respectively. As seen in Fig. S15 of the SM [20], the three periodicities are significant up to at least $20 \mathrm{GV}$. Note that both the unnormalized power of these periodicities and the flux variance in the two time intervals decrease with increasing 


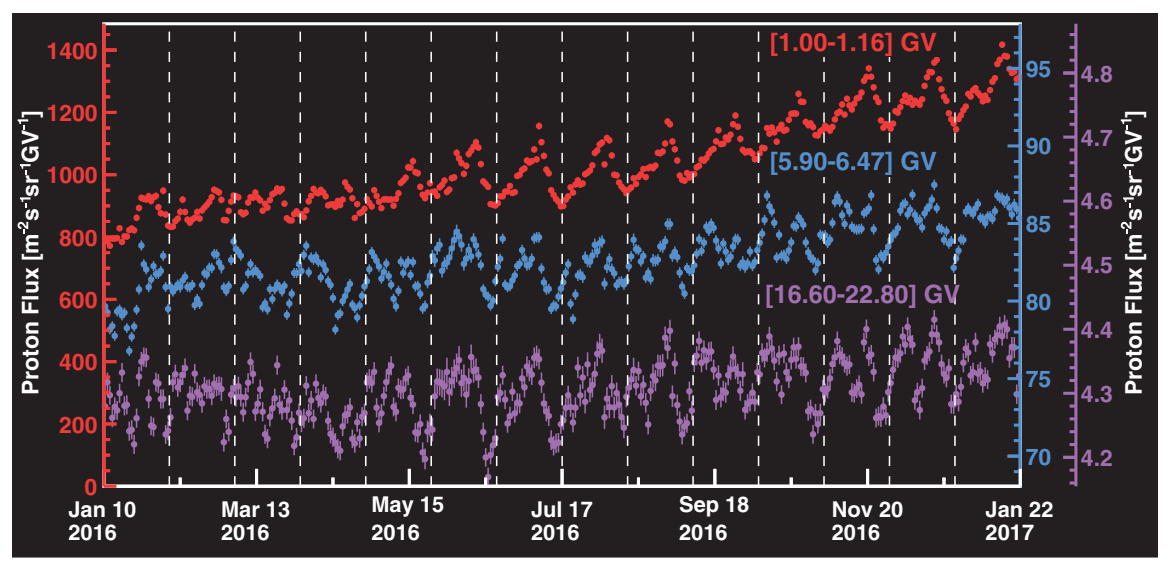

FIG. 2. The daily proton fluxes measured in 2016 for three rigidity bins. Vertical dashed lines separate Bartels rotations. As seen, double-peak and triple-peak structures are visible in different Bartels rotations.

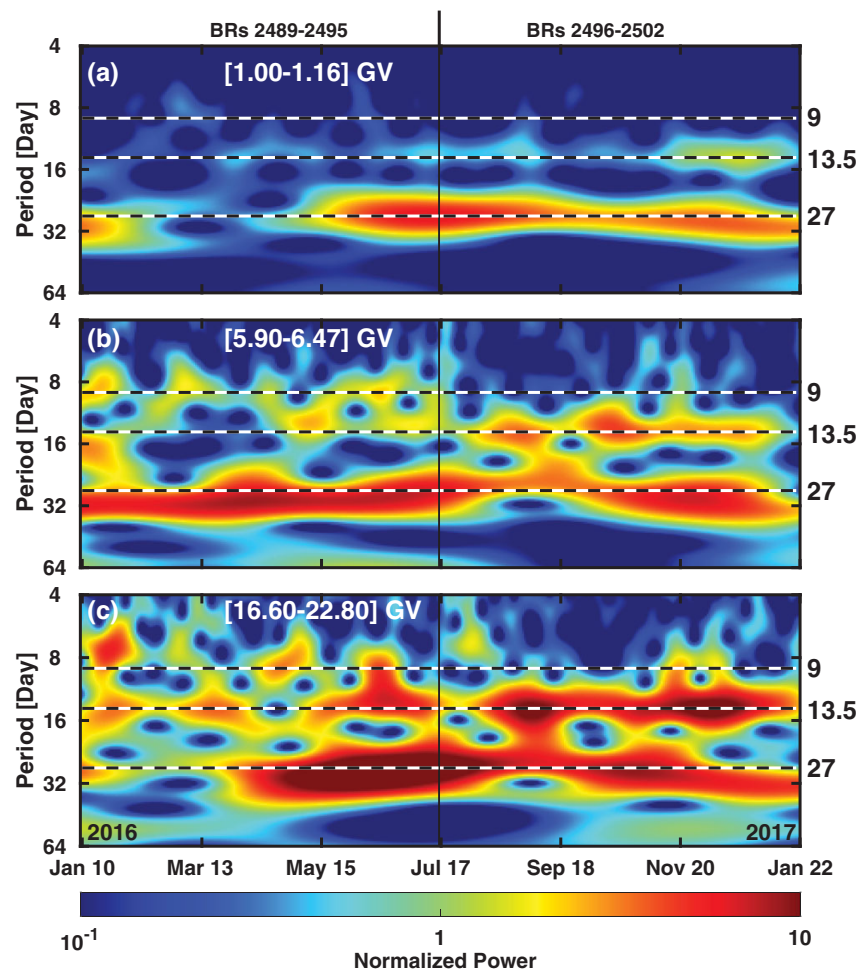

FIG. 3. The wavelet time-frequency power spectrum of daily AMS proton fluxes from January 10, 2016 to January 21, 2017 for three rigidity bins (a) [1.00-1.16] GV, (b) [5.90-6.47] GV, and (c) [16.60-22.80] GV. The color code indicates the normalized power. The vertical scales are in decreasing period (increasing frequency). As seen, periods of 9, 13.5, and 27 days are observed. The strength of all three periodicities changes with time and rigidity. In particular, shorter periods of 9 and 13.5 days, when present, are more visible at $6 \mathrm{GV}$ and $20 \mathrm{GV}$ compared to $1 \mathrm{GV}$. The horizontal dashed lines indicate the locations of 9-day, 13.5day, and 27-day periods shown on the right scale. The vertical solid line indicates the boundary of the two time intervals marked on the top. Above $5.90 \mathrm{GV}$, the first time interval (BRs 24892495 ) is when the 9-day period is visible; the second time interval (BRs 2496-2502) is when the 9-day period is not visible. rigidity as shown in Fig. S16 of the SM [20]. The peak values of the normalized power around 27 days as a function of rigidity for each year are shown in Fig. S17 of the SM [20]. As seen, the 27-day periodicity only becomes significant from 2014, and its rigidity dependence varies in different time intervals and is different from the rigidity dependences of 9-day and 13.5-day periods.

The intensity variations of cosmic rays are caused by the temporal evolution of the interplanetary space environment. In particular, the solar wind speed is related to cosmic-ray advection, the variation of solar wind proton density is related to cosmic-ray adiabatic energy changes, and the interplanetary magnetic field is related to cosmic-ray diffusion and drifts [1]. Figure S18 of the SM [20] shows the wavelet time-frequency power spectra of the daily averages of these interplanetary space environment properties [38] in 2016. To investigate their relations with the observed periodicities in the proton fluxes, the cross wavelet transform [37] as described in SM [20] is performed as shown in Fig. S19 of the SM [20]. The proton fluxes are observed to be related to the interplanetary space environment properties for all periodicities, such as the radial component (along the Sun-Earth direction) of the interplanetary magnetic field for the 9-day periodicity and the solar wind speed for the 13.5-day periodicity.

In conclusion, we have presented the precision measurements of the daily proton fluxes in cosmic rays from $1 \mathrm{GV}$ to $100 \mathrm{GV}$ between May 20, 2011 and October 29, 2019 based on $5.5 \times 10^{9}$ protons. The proton fluxes exhibit variations on different timescales, in days, months, and years. From 2014 to 2018, we observed recurrent flux variations with a period of 27 days. Shorter periods of 9 days and 13.5 days are observed in 2016. The strength of all three periodicities changes with both time and rigidity. Unexpectedly, the strength of 9-day and 13.5-day periodicities increases with increasing rigidities up to $\sim 10 \mathrm{GV}$ and $\sim 20 \mathrm{GV}$ respectively. Then the strength of the periodicities decreases with increasing rigidity up to $100 \mathrm{GV}$. 


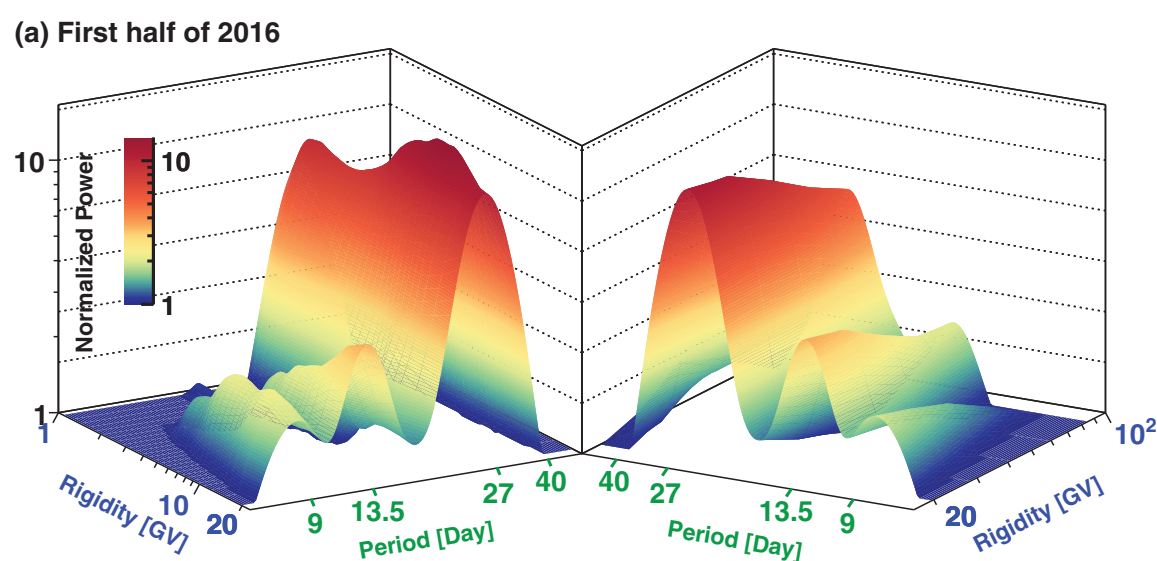

(b) Second half of 2016

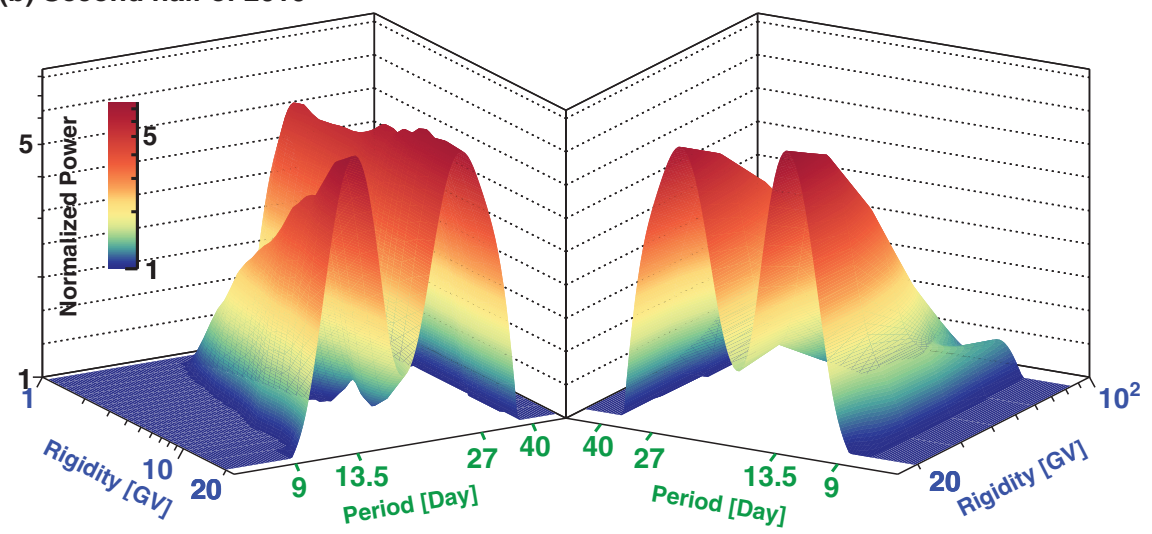

FIG. 4. The normalized power as a function of rigidity and period for (a) the first and (b) the second half of 2016 from 1 to $20 \mathrm{GV}$ and from 20 to $100 \mathrm{GV}$. As seen, the strength of 9-day, 13.5-day, and 27-day periodicities is rigidity dependent. In particular, the strength of 9-day and 13.5-day periodicities increases with increasing rigidity up to $\sim 10 \mathrm{GV}$ and $\sim 20 \mathrm{GV}$, respectively, and then decreases with increasing rigidity up to $100 \mathrm{GV}$.

These new precision measurements provide unique inputs to the understanding of cosmic rays in the heliosphere.

We are grateful for important physics discussions with Jonathan Feng and Igor Moskalenko. We thank former NASA Administrator Daniel S. Goldin for his dedication to the legacy of the ISS as a scientific laboratory and his decision for NASA to fly AMS as a DOE payload. We also acknowledge the continuous support of the NASA leadership, particularly William H. Gerstenmaier, and of the Johnson Space Center (JSC) and Marshall Space Flight Center (MSFC) flight control teams that have allowed AMS to operate optimally on the ISS for ten years. We are grateful for the support of Jim Siegrist, Glen Crawford, and their staff of the DOE including resources from the National Energy Research Scientific Computing Center under Contract No. DE-AC02-05CH11231. We gratefully acknowledge the strong support from CERN including Fabiola Gianotti, and the CERN IT department including Bernd Panzer-Steindel, and from the European Space Agency including Johann-Dietrich Wörner and Simonetta Di Pippo. We also acknowledge the continuous support from Massachusetts Institute of Technology (MIT) and its School of Science, Michael Sipser, and the Laboratory for Nuclear Science, Boleslaw Wyslouch. Research supported by: Chinese Academy of Sciences, Institute of High Energy Physics, Institute of Electrical Engineering, China Academy of Space Technology, National Natural Science Foundation, and Ministry of Science and Technology, the China Scholarship Council, the provincial governments of Shandong, Jiangsu, Guangdong, Shandong University, and the Shandong Institute of Advanced Technology, China; the Academy of Finland, Project No. 321882, Finland; CNRS/ IN2P3 and CNES, France; DLR under Grants No. 50001805 and computing support on the JARA Partition of the RWTH Aachen supercomputer, Germany; INFN and ASI under ASI-INFN Agreements No. 2014-037R.1-2017 and No. 2019-19-HH.0 and ASI-University of Perugia Agreement No. 2019-2-HH.0, Italy; CHEP and NRF under Grant No. NRF-2018R1A6A1A06024970 at Kyungpook National University, Korea; the Consejo Nacional de Ciencia y Tecnología and UNAM, Mexico; NWO under Grant No. 680-1-004, Netherlands; FCT under Grant No. CERN/FIS-PAR/0013/2019, Portugal; the Ministry of Science and Higher Education under Project 
No. 0723-2020-0040, Russia; CIEMAT, IAC, CDTI, and SEIDI-MINECO under Grants No. PID2019-107988 GBC21/C22, No. CEX2019-000920-S, and No. MDM-20150509, Spain; the Swiss National Science Foundation (SNSF), federal and cantonal authorities, and the Fondation Dr. Manfred Steuer, Switzerland; Academia Sinica and the Ministry of Science and Technology (MOST) under Grants No. 107-2119-M-006-015-MY3, No. 109-2112-M001-029, and No. CDA-105-M06, former Presidents of Academia Sinica Yuan-Tseh Lee and Chi-Huey Wong and former Ministers of MOST Maw-Kuen Wu and LuoChuan Lee, Taiwan; the Turkish Energy, Nuclear and Mineral Research Agency (TENMAK) under Grants No. 2020TAEK (CERN)A5.H1.F5-26, Turkey; and NSF Grants No. 1455202 and No. 2013228, Wyle Laboratories Grant No. 2014/ T72497, and NASA NESSF Grant No. HELIO15F0005, USA.

*Also at Nikhef, 1098 XG Amsterdam, Netherlands.

${ }^{\dagger}$ Also at ASI Space Science Data Center (SSDC), 00133 Roma, Italy.

"Also at Policlinico S. Orsola-Malpighi, 40138 Bologna, Italy.

[1] E. N. Parker, The passage of energetic charged particles through interplanetary space, Planet. Space Sci. 13, 9 (1965).

[2] M. S. Potgieter, Solar modulation of cosmic rays, Living Rev. Solar Phys. 10, 3 (2013).

[3] D. H. Hathaway, The solar cycle, Living Rev. Solar Phys. 12, 4 (2015).

[4] Sunspot data from the World Data Center SILSO, Royal Observatory of Belgium, Brussels at http://www.sidc.be/ silso/cyclesminmax.

[5] M. Zhang, Modulation of galactic cosmic rays at solar maximum: Observations, Adv. Space Res. 32, 603 (2003); H. V. Cane, Coronal mass ejections and Forbush decreases, Space Sci. Rev. 93, 55 (2000); M. S. Potgieter, J. A. Le Roux, L.F. Burlaga, and F. B. McDonald, The role of merged interaction regions and drifts in the heliospheric modulation of cosmic rays beyond $20 \mathrm{AU}$ : A computer simulation, Astrophys. J. 403, 760 (1993); G. Wibberenz, I. G. Richardson, and H. V. Cane, A simple concept for modeling cosmic ray modulation in the inner heliosphere during solar cycles 2023, J. Geophys. Res. 107, 1353 (2002).

[6] J. A. Simpson, A brief history of recurrent solar modulation of the galactic cosmic rays (1937-1990), Space Sci. Rev. 83, 169 (1998).

[7] C. Paizis et al., Amplitude evolution and rigidity dependence of the 26-day recurrent cosmic ray decreases: COSPIN/ KET results, J. Geophys. Res. 104, 28241 (1999).

[8] Z. Shen, G. Qin, P. Zuo, F. Wei, and X. Xu, A study of variations of galactic cosmic-ray intensity based on a hybrid data-processing method, Astrophys. J. 900, 143 (2020).

[9] R. Modzelewska et al., Study of the 27 day variations in GCR fluxes during 2007-2008 based on PAMELA and ARINA observations, Astrophys. J. 904, 3 (2020), In this paper, the rigidity dependence of the 27-day periodicity in 2007-2008 has been directly measured.

[10] R. Modzelewska and A. Gil, Recurrence of galactic cosmicray intensity and anisotropy in solar minima $23 / 24$ and 24/25 observed by ACE/CRIS, STEREO, SOHO/EPHIN and neutron monitors, Astron. Astrophys. 646, A128 (2021).

[11] A. López-Comazzi and J. J. Blanco, Short-term periodicities observed in neutron monitor counting rates, Sol. Phys. 295, 81 (2020).

[12] M. Armano et al., Characteristics and energy dependence of recurrent galactic cosmic-ray flux depressions and of a Forbush decrease with LISA pathfinder, Astrophys. J. 854, 113 (2018).

[13] I. Sabbah and K. Kudela, Third harmonic of the 27 day periodicity of galactic cosmic rays: Coupling with interplanetary parameters, J. Geophys. Res. 116, A04103 (2011).

[14] M. Temmer, B. Vršnak, and A. M. Veronig, Periodic appearance of coronal holes and the related variation of solar wind parameters, Sol. Phys. 241, 371 (2007).

[15] S. E. Forbush, On cosmic-ray effects associated with magnetic storms, Terr. Magn. Atmos. Electr. 43, 203 (1938).

[16] A. Gil and M. V. Alania, Theoretical and experimental studies of the rigidity spectrum of the 27-day variation of the galactic cosmic ray intensity in different epochs of solar activity, Sol. Phys. 283, 565 (2013).

[17] M. Aguilar et al., Observation of Fine Time Structures in the Cosmic Proton and Helium Fluxes with the Alpha Magnetic Spectrometer on the International Space Station, Phys. Rev. Lett. 121, 051101 (2018).

[18] M. Aguilar et al., The Alpha Magnetic Spectrometer (AMS) on the international space station: Part II results from the first seven years, Phys. Rep. 894, 1 (2021).

[19] A. Kounine, The alpha magnetic spectrometer on the international space station, Int. J. Mod. Phys. E 21, 1230005 (2012); S. Rosier-Lees, New results from AMS, in Proceedings of Astroparticle Physics TEVPA/IDM, Amsterdam, 2014 (unpublished); S. Ting, The Alpha Magnetic Spectrometer on the international space station, Nucl. Phys. B, Proc. Suppl. 243-244, 12 (2013); S.-C. Lee, Latest results from AMS, in Proceedings of the 20th International Conference on Supersymmetry and Unification of Fundamental Interactions (SUSY 2012), Beijing, 2012 (unpublished); M. Aguilar, The AMS experiment on the ISS, in Proceedings of the XL International Meeting on Fundamental Physics, Centro de Ciencias de Benasque Pedro Pascual, 2012 (unpublished); S. Schael, Status of the AMS02 experiment on the ISS, in Proceedings of the 10th Symposium on Sources and Detection of Dark Matter and Dark Energy in the Universe, Los Angeles, 2012 (unpublished); B. Bertucci, The AMS-02 detector operation in space, Proc. Sci. EPS-HEP2011 (2011) 67; M. Incagli, Astroparticle physiscs with AMS02, AIP Conf. Proc. 1223, 43 (2010); R. Battiston, The antimatter spectrometer (AMS02): A particle physics detector in space, Nucl. Instrum. Methods Phys. Res., Sect. A 588, 227 (2008).

[20] See Supplemental Materials at http://link.aps.org/ supplemental/10.1103/PhysRevLett.127.271102 for the AMS detector description, details of event selection, details of wavelet analysis, figures, and the tabulated daily proton 
fluxes as functions of rigidity.Note that the data can also be downloaded in different formats from the AMS website https://ams02.space/, the ASI cosmic-ray database at https:// tools.asdc.asi.it/CosmicRays, and the LPSC cosmic-day database at https://lpsc.in2p3.fr/crdb/.

[21] K. Lübelsmeyer et al., Upgrade of the Alpha Magnetic Spectrometer (AMS-02) for long term operation on the international space station (ISS), Nucl. Instrum. Methods Phys. Res., Sect. A 654, 639 (2011).

[22] B. Alpat et al., The internal alignment and position resolution of the AMS-02 silicon tracker determined with cosmic-ray muons, Nucl. Instrum. Methods Phys. Res., Sect. A 613, 207 (2010).

[23] G. Ambrosi, V. Choutko, C. Delgado, A. Oliva, Q. Yan, and Y. Li, The spatial resolution of the silicon tracker of the Alpha Magnetic Spectrometer, Nucl. Instrum. Methods Phys. Res., Sect. A 869, 29 (2017).

[24] Y. Jia, Q. Yan, V. Choutko, H. Liu, and A. Oliva, Nuclei charge measurement by the Alpha Magnetic Spectrometer silicon tracker, Nucl. Instrum. Methods Phys. Res., Sect. A 972, 164169 (2020).

[25] V. Bindi et al., Calibration and performance of the AMS-02 time of flight detector in space, Nucl. Instrum. Methods Phys. Res., Sect. A 743, 22 (2014).

[26] J. Allison et al., Recent developments in GEANT4, Nucl. Instrum. Methods Phys. Res., Sect. A 835, 186 (2016); J. Allison et al., GEANT4 developments and applications, IEEE Trans. Nucl. Sci. 53, 270 (2006); S. Agostinelli et al., GEANT4-a simulation toolkit, Nucl. Instrum. Methods Phys. Res., Sect. A 506, 250 (2003).

[27] Q. Yan, V. Choutko, A. Oliva, and M. Paniccia, Measurements of nuclear interaction cross sections with the Alpha Magnetic Spectrometer on the international space station, Nucl. Phys. A996, 121712 (2020).

[28] M. Aguilar et al., Precision Measurement of the Proton Flux in Primary Cosmic Rays from Rigidity 1 GV to 1.8 TV with the Alpha Magnetic Spectrometer on the International Space Station, Phys. Rev. Lett. 114, 171103 (2015).
[29] M. Aguilar et al. (AMS Collaboration), Measurement of the geomagnetic cutoff with the alpha magnetic spectrometer on the international space station (to be published).

[30] C. C. Finlay et al., International geomagnetic reference field: The eleventh generation, Geophys. J. Int. 183, 1216 (2010); E. Thébault et al., International geomagnetic reference field: The 12th generation, Earth Planets Space 67, 79 (2015); Geomagnetic Field Modeling Working Group, IGRF-13 model (2019), https://www.ngdc.noaa.gov/IAGA/ vmod/igrf.html.

[31] P. Bobik, G. Boella, M. J. Boschini, D. Grandi, M. Gervasi, K. Kudela, S. Pensotti, and P. G. Rancoita, Magnetospheric transmission function approach to disentangle primary from secondary cosmic ray fluxes in the penumbra region, J. Geophys. Res. 111, A05205 (2006); N. A. Tsyganenko and M. I. Sitnov, Modeling the dynamics of the inner magnetosphere during strong geomagnetic storms, J. Geophys. Res. 110, A03208 (2005).

[32] M. Aguilar et al. (AMS Collaboration), Measurement of the Deuteron Flux with the Alpha Magnetic Spectrometer on the International Space Station (to be published).

[33] J. Berdugo, V. Choutko, C. Delgado, and Q. Yan, Determination of the rigidity scale of the Alpha Magnetic Spectrometer, Nucl. Instrum. Methods Phys. Res., Sect. A 869, 10 (2017).

[34] M. Aguilar et al. (AMS Collaboration), Measurement of the Solar Energetic Particles with the Alpha Magnetic Spectrometer on the International Space Station (to be published).

[35] O. Adriani et al., Unexpected cyclic behavior in cosmic-ray protons observed by PAMELA at 1 au, Astrophys. J. Lett. 852, L28 (2018), We thank Prof. Piergiorgio Picozza for providing the PAMELA data in digital format to us.

[36] C. Torrence and G. P. Compo, A practical guide to wavelet analysis, Bull. Am. Meteorol. Soc. 79, 61 (1998).

[37] A. Grinsted, J. C. Moore, and S. Jevrejeva, Application of the cross wavelet transform and wavelet coherence to geophysical time series, Nonlinear Processes Geophys. 11, 561 (2004).

[38] NASA/GSFC's OMNI dataset at daily resolution: https:// omniweb.gsfc.nasa.gov/. 